\title{
Análisis del desarrollo de la alianza terapéutica en Musicoterapia a través del discurso musical
}

\author{
Jalil Majul Villarreal ${ }^{1}$ \\ José Fernando Fernández Company²
}

Recibido: 20/02/2020

Aceptado: 21/04/2020

RESUMEN. Los objetivos de esta investigación se centran en averiguar si surge la alianza terapéutica entre paciente y terapeuta durante un tratamiento de musicoterapia determinado y en proponer una metodología apropiada para el análisis de intervenciones de este tipo. El objetivo de la intervención terapéutica es el de reducir la frecuencia de conductas obsesivas y mejorar la atención. Se han grabado las sesiones y se han codificado las conductas que configuran la alianza terapéutica para obtener datos y analizarlos. El resultado de los estadísticos descriptivos sugiere que es posible crear una relación terapéutica con el tratamiento de musicoterapia propuesto. En conclusión, se demuestra la posibilidad de surgimiento de la alianza terapéutica en musicoterapia, a pesar de prescindir del lenguaje verbal y se abre una línea de investigación.

Palabras clave: Musicoterapia, Alianza Terapéutica, Síndrome de Down, Comunicación, Música.

\section{Analysis of the development of the therapeutic alliance in Music therapy through musical language}

ABSTRACT. The objectives of this research are to find out if the therapeutic alliance between the patient and the therapist arises during a specific music therapy treatment and propose a specific methodology for the analysis of interventions of this type. The goal of therapeutic intervention is to reduce the frequency of obsessive behaviors and improve attention. The sessions have been recorded and the behaviors that configure the therapeutic alliance to obtain data and analyze them have been codified. The result of the suggested descriptive statistics is possible to create a therapeutic relationship with the proposed music therapy treatment. In conclusion, the possibility of surgery of the therapeutic alliance in music therapy, a prescription weight of verbal language is demonstrated and a line of investigation is opened.

Keywords: Music Therapy, Therapeutic Alliance, Down Syndrome, Communication, Music.

Sumario. 1. Introducción.2. Método.3. Resultados 4. Discusión 5. Conclusiones 6. Referencias

Cómo citar. Majul, J. y Fernández, J. F. (2019). Análisis del desarrollo de la alianza terapéutica en Musicoterapia a través del discurso musical. Revista de Investigación en Musicoterapia, 3, 72-85 https://doi.org/10.15366/rim2019.3.005

\footnotetext{
${ }^{1}$ Máster Musicoterapia Avanzada y Aplicaciones Universidad Autónoma de Madrid

${ }^{2}$ Máster Musicoterapia Avanzada y Aplicaciones Universidad Autónoma de Madrid
} 


\section{INTRODUCCIÓN}

Se ha propuesto este hilo de investigación con el fin de ahondar sobre los aspectos clave en la comunicación a través de la música, dentro de las sesiones de musicoterapia. Existe una escasa línea de investigación que relacione el concepto de alianza terapéutica y musicoterapia. Resulta muy importante dar luz a este tema porque se puede caer en el error de dar por supuesto como viable el papel comunicativo de la música a lo largo del proceso terapéutico independientemente de su duración.

La música en musicoterapia sustituye al lenguaje verbal y escrito como forma de comunicación y expresión central facilitando la creación de una relación terapéutica a pesar de no compartir las características comunicativas existentes en el desarrollo de las técnicas convencionales.

La presente investigación tiene como objetivo saber si es posible establecer una relación terapéutica en un proceso compuesto por 6 sesiones de musicoterapia a pesar de que las técnicas utilizadas se centran en la música y no en el lenguaje.

Los objetivos del tratamiento diseñado para el varón son: el aumento de la expresión emocional, control motor fino, disminuir los niveles de activación y reducir la frecuencia de las conductas obsesivas. Los objetivos del tratamiento para la mujer son: conseguir una apertura de la boca más pronunciada, reducir el bruxismo durante las sesiones, aumentar el tiempo de atención durante las tareas, incrementar expresión emocional y corporal y mejorar la postura del cuello y de la espalda a través del contacto visual y la mirada dirigida hacia la parte superior de su campo visual.

Existen distintos tipos de terapias orientadas al tratamiento psicológico de las personas, aunque el más extendido en la actualidad es el modelo cognitivo conductual, que presenta una serie de procedimientos estructurados y organizados en tres generaciones de terapia (Ruiz, Díaz y Villalobos, 2012). Si se pone el foco en las principales terapias de corte cognitivo conductual de segunda generación, como la Terapia Racional Emotiva (Ellis y Grieger, 1990), Terapia Cognitiva de Beck (Beck y Alford, 2009) o el Entrenamiento en Inoculación del Estrés (Meichenbaum, 1985), se puede comprobar que la herramienta principal para la intervención es la comunicación verbal porque el desarrollo de este tipo de intervenciones se llevan a cabo a través del discurso.

Como se ha podido comprobar, el lenguaje verbal es un elemento central en muchos tipos de terapias convencionales, por tanto, esta herramienta se vuelve imprescindible para llevar a 
cabo las técnicas pertenecientes en las intervenciones anteriormente citadas. Existen algunos colectivos que encuentran barreras en la producción y comprensión del lenguaje verbal y escrito (Bello, Onofrio y Caselli, 2014). Las personas con Síndrome de Down (SD) tienen grandes dificultades en el área de la comprensión lectora (Carr, 1995) y se ha demostrado que esta está correlacionada con la habilidad lingüística, de modo que, los déficits de la comprensión lectora en personas con SD son el resultado de dificultades implícitas en el lenguaje (Nash y Heath, 2011). Cabría pensar que las dificultades con el lenguaje de pacientes con SD residen en la discriminación fonológica, pero la realidad es que están más relacionadas con la memoria verbal a corto plazo (Purser y Jarrold, 2012). A pesar de las dificultades en la comprensión lectora de las personas con $\mathrm{SD}$, esta presenta ventajas respecto a la comprensión verbal (Roch, Florit y Levorato, 2011). En el SD las habilidades lingüísticas se ven más afectadas que las habilidades no verbales (Laws y Bishop, 2003), por tanto, el lenguaje no verbal en terapia adquiere un papel más relevante en aras de la comunicación.

El rapport o alianza terapéutica se entiende como un elemento sin el cual no habría terapia exitosa (Roth y Fonagy, 2005). También está definida como la base de un proceso terapéutico en la que el paciente y el terapeuta se vuelven aliados para combatir la enfermedad (Michel, 2011) y hoy en día se considera un prerrequisito para la aplicación de técnicas provenientes de la psicología cognitivo conductual (Beck, Rush, Shaw, y Emery, 1979) e incluso en la farmacoterapia (Krupnick, Sotsky, Elkin, Watkins y Pilkonis, 1996). El término de relación terapéutica o rapport fue advertido y señalado por Freud (1955) a través de los mecanismos de transferencia. Un factor importante para el desarrollo de la alianza terapéutica es el tiempo entre sesiones (Hartmann, Orlinsky y Zeeck, 2011), aunque lo más importante es el tiempo de sesión. La herramienta central de las sesiones de terapia convencionales como el psicoanálisis y la terapia cognitivo conductual es el lenguaje oral y escrito, por tanto, se convierte en un elemento decisivo para la creación de la relación terapéutica.

Al ser un hecho que las personas con SD presentan barreras en la comunicación verbal, cabría preguntarse qué tipos de terapias utilizan menos recursos verbales en sus técnicas como piedra angular, con el fin de llevar a cabo una intervención idónea, que favorezca el desarrollo del rapport o alianza terapéutica y prescinda del lenguaje verbal o escrito en la medida de lo posible utilizando otras formas de comunicación como la música.

La empatía y la comprensión se basan en el discurso musical y no en el discurso verbal. Las experiencias que se comparten en musicoterapia se comparten a través de la música y en pequeña medida a través del discurso. Por ejemplo, en una improvisación la comunicación y Revista de Investigación en Musicoterapia, 3, 2019, pp. $72-85$ 
la empatía se traducen en una pieza musical improvisada o establecida en la que el musicoterapeuta y el paciente van andando juntos hacia un objetivo común sin necesidad de expresar verbalmente lo que está sucediendo. El buen musicoterapeuta entiende la expresión musical y emocional de un paciente y lo acompaña de forma coherente, siendo este el momento en el que se lleva a cabo una conducta empática haciendo uso de la música como forma de comunicación.

La música, además de ser un arte, es considerada un lenguaje que comunica a través de la combinación de sonidos; creando estructuras armónicas que expresan ideas y sentimientos (Gutiérrez, 2013). No solo se ha definido a la música como una forma de comunicación, sino que se ha calificado, incluso, como un elemento expresivo subyacente y necesario del lenguaje verbal al señalar los atributos musicales y la función comunicativa de las vocales (Robson, 1959). En esta línea de investigación se ha trabajado sobre la importancia de la prosodia en el habla para distinguir emociones y su relación con las curvas de sonido y la musicalidad (Rigoulot, Wassiliwizky y Pell, 2013). La música, además de ser una forma de comunicación, también es un medio independiente del habla que comunica emociones (Spreckelmeyer, Altenmüller, Colonius y Münte, 2013) y puede influir en el humor, la conducta, la interacción social y el desempeño (Chavin, 2002). Se especula que la música tiene una vía de comunicación única con el cerebro (Flaig y Large, 2014) aunque esta se encuentra relacionada con las redes neuronales más importantes para el procesamiento del lenguaje por la cercanía y uso compartido.

La música y el lenguaje poseen una característica común; son sistemas cognitivos divididos en distintos niveles de procesamiento (Poeppel y Hickok, 2004), además, la memoria musical y la memoria verbal son procesadas por redes neuronales distintas, pero muy próximas una de otra (Groussard, Rauchs, Landeau, Viader, Desgranges, Eustache y Platel, 2010). Algunos autores sostienen que la música y el lenguaje se procesan con una red neuronal común abarcando el procesamiento melódico, léxico y fonológico (Schön, Gordon, Campagne, Magne, Astésano, Anton y Besson, 2010). Cuando se canta con letra se activan notoriamente redes neuronales del hemisferio izquierdo (Wilson, 2011), que es donde se encuentran las áreas de Broca y Wernicke, implicadas en el procesamiento del lenguaje. El lóbulo temporal derecho está implicado en las habilidades musicales (Hyde, Peretz y Zatorre, 2008) por tanto, se puede asumir que la música se procesa en distintas áreas y estructuras. Si la música conforma un medio de comunicación, es posible que su introducción en los tratamientos tenga algún efecto sobre la socialización en distintos ámbitos. Atendiendo al nivel Revista de Investigación en Musicoterapia, 3, 2019, pp. $72-85$ 
comunicativo de los pacientes Toll afirma que, en centros de día y residencias, aumenta cuando la música forma parte del tratamiento; además, incrementa la socialización con otros pacientes y cuidadores (Toll, 2007).

Resulta de gran interés saber si en las sesiones de musicoterapia propuestas se genera una relación terapéutica con el uso de la música como herramienta central de la terapia, porque cuánto más fuerte es la alianza terapéutica (AT), más cambio terapéutico se prevé (Orlinsky, Ronnestad y Willutzki, 2004). El desarrollo de la AT no solo es un predictor de éxito, también es un elemento inherente del asesoramiento al paciente (Warlick, Richter, Catley, Gajewski, Martin y Mussulman, 2018).

Se puede evaluar la AT con instrumentos como el Helping Alliance Questionnaire (HAQ), que está diseñado para terapias psicodinámicas cortas (Bassler y Nübling, 2015) y otras versiones de este mismo test (Alexander y Luborsky, 1986). Existe otro instrumento para evaluar la AT; Working Alliance Inventory (WAI) (Horvath y Greenberg, 1986). Este instrumento ha sido validado para la población española dando lugar al WAI-S (AndradeGonzález y Fernández-Liria, 2015), que cuenta con una escala que debe ser respondida por el terapeuta. Los instrumentos anteriores se derivan de la definición tripartita de AT de Bordin (Summers y Barber, 2003). Actualmente, no han sido validado instrumentos relacionados con la AT para pacientes con SD, por tanto, es importante dar más peso a la definición de Bordin, que señala tres componentes interdependientes de la AT; aceptación de los objetivos, aceptación de las técnicas a utilizar y creación de un lazo afectivo (Bordin, 1979) expresado a través del agrado, confianza y respeto (Horvath, 1994). La calidad de la AT dependerá del grado de acuerdo que haya entre paciente y terapeuta en relación a las tareas y objetivos del tratamiento, utilizando el lazo afectivo como mediador (Bordin, 1979).

\section{MÉTODO}

\section{PARTICIPANTES}

En esta investigación participan dos con SD, un hombre y una mujer de edad adulta. La mujer, AM, padece de bruxismo, la postura de su espalda es encorvada, su línea de visión no suele superar la altura de su nariz, le cuesta mantener la atención y a penas abre la boca cuando habla y esto dificulta la comunicación con los trabajadores del centro y con sus compañeros. El varón, $\mathrm{N}$, tiene dificultades para permanecer tranquilo, tiene conductas obsesivas relacionadas con ajustar los cordones y el cinturón, repite constantemente hechos 
ocurridos en días anteriores y se le dificulta seguir las indicaciones de los cuidadores. Los participantes viven y acuden a musicoterapia en la residencia y centro de día Carmen Sevilla, en Madrid. No se ha informado de ninguna condición de salud especial por parte de los cuidadores durante la realización de la investigación.

\section{Procedimiento}

\section{Investigación}

Se llevará a cabo una intervención con sesiones estructuradas grabadas en vídeo y se realizará un posterior análisis cuantitativo de conductas target para medir la existencia de una alianza terapéutica. Las conductas se medirán en dos momentos diferentes de la intervención: en la sesión inicial y en la penúltima sesión del proceso terapéutico.

Se utilizará un teléfono móvil para grabar las sesiones con el fin de analizar posteriormente el material resultante con el ATLAS.ti 8. Con este software se codificarán las conductas target $\mathrm{y}$, a continuación, se procesarán de forma cuantitativa con el objetivo llevar a cabo un análisis estadístico. Se hará uso del Microsoft Office Excel 2007 para alojar y organizar los datos procedentes del ATLAS.ti 7. El análisis estadístico se llevará a cabo con el IBM SPSS Statistics 23 haciendo un análisis estadístico no paramétrico de Wilcoxon para comparar las medias obtenidas de las puntuaciones iniciales y las finales.

Se medirá el desarrollo de la AT a lo largo del tratamiento utilizando el WAI-T; subescala dirigida al terapeuta (Anexo 2) perteneciente al instrumento WAI validado a la población española (Andrade-González y Fernández-Liria, 2015) y comparando los resultados obtenidos de la primera y última sesión, es decir, el número de veces que aparecen conductas target favorables a la AT. Las conductas a tener en cuenta están basadas en la definición tripartita de Alianza terapéutica de Bordin (1979). Las conductas target generadas se encuentran agrupadas dentro de los tres elementos de la definición como se muestra en la Tabla 1. 
Tabla 1

Conductas target de la AT

Alianza Terapéutica

\begin{tabular}{|c|c|c|c|}
\hline & $\begin{array}{l}\text { Aceptación de los } \\
\text { objetivos }\end{array}$ & $\begin{array}{l}\text { Aceptación de las técnicas a } \\
\text { utilizar }\end{array}$ & $\begin{array}{l}\text { Creación de un lazo } \\
\text { afectivo }\end{array}$ \\
\hline Conductas & $\begin{array}{l}\text { - El paciente hace el } \\
\text { esfuerzo de hacer el } \\
\text { ejercicio que se le pide, } \\
\text { aunque sus barreras } \\
\text { como la pérdida de } \\
\text { concentración o falta de } \\
\text { movilidad representen } \\
\text { dificultades. } \\
\text { - Verbalizar o mostrar } \\
\text { conciencia sobre la } \\
\text { problemática } \\
\text { paciente. }\end{array}$ & $\begin{array}{l}\text { - El paciente presenta } \\
\text { voluntad para comenzar a } \\
\text { trabajar el ejercicio que se } \\
\text { le pide. } \\
\text { - Declaraciones de agrado } \\
\text { acerca de los ejercicios. } \\
\text { - Sonrisas o risa respecto a la } \\
\text { actividad. } \\
\text { - Prestar atención a las } \\
\text { instrucciones. } \\
\text { - Persistencia en la tarea. }\end{array}$ & $\begin{array}{l}\text { - Surge la risa en las } \\
\text { sesiones. } \\
\text { - El paciente se deja guiar } \\
\text { por el terapeuta. } \\
\text { - Demostraciones de } \\
\text { afecto por parte del } \\
\text { paciente. } \\
\text { - Reducción de las } \\
\text { conductas de vergüenza. } \\
\text { - Contacto visual. }\end{array}$ \\
\hline
\end{tabular}

\section{Sesiones}

Las sesiones para $\mathrm{N}$ tienen la siguiente estructura:

- Pasaje de bienvenida (Anexo 3): Se toca un pasaje de bienvenida utilizando la guitarra y la voz y se le pide al paciente que salude con un "hola" de vuelta cuando se le salude.

- Respiración: Se han compuesto y grabado tres pasajes (Anexo 4) con distinto tempo en función de las habilidades en respiración del paciente y su capacidad pulmonar (Anexo 4.2). Se pide al paciente que respire y expire cuando se le indique en función de las frases musicales. Esta técnica se utiliza para reducir la activación y mejorar la concentración (Vázquez, 2001).

- Movimiento a través de tres ejercicios: 
- Baile: Con percusión y melodía vocal se toca el Waltz Sobre las Olas de Juventino Rosas para trabajar la intimidad, el movimiento, la complicidad y evocar la risa haciendo movimientos exagerados.

- Movimiento con maracas: Se entrega una maraca y se toca un blues clásico de doce compases y se le cantan las indicaciones referidas a la orientación y lugar en el que se tiene que tocar el huevo. Este ejercicio favorece el movimiento de los brazos y trabaja la atención sostenida.

- Movimiento con el cuerpo: Se toca un blues de doce compases y se cantan las instrucciones referidas a una parte del cuerpo y el tipo de movimiento requerido cada dos o cuatro compases.

- Improvisación con instrumento: Se realiza una improvisación de forma libre con cualquier instrumento que esté disponible. Este ejercicio está basado en el modelo de improvisación libre de Alvin (1975). Se procura utilizar en algún momento de la improvisación instrumentos que requieran del uso de la motricidad fina, como los crótalos.

- Interpretación de una canción: Se interpreta a dúo una canción conocida por el paciente o sugerida por el musicoterapeuta.

- Improvisación vocal: Paciente y terapeuta improvisan con la voz. La forma musical resultante de la improvisación va en función del estado de ánimo del paciente y lo que este intenta expresar.

- Pasaje de despedida (Anexo 5): Se interpreta la canción de despedida. En la parte final de la canción el paciente tiene que estar atento para decir adiós dos veces.

Las sesiones para AM tienen la siguiente estructura:

- Pasaje de bienvenida (Anexo 3): Se toca un pasaje de bienvenida utilizando la guitarra y la voz y se le pide a la paciente que salude con un "hola" de vuelta cuando se le salude.

- Respiración: Se han compuesto y grabado tres pasajes (Anexo 4) con distinto tempo en función de las habilidades en respiración de la paciente y su capacidad pulmonar 
(Anexo 4.3). Se pide a la paciente que respire y expire cuando se le indique en función de las frases musicales.

- Movimiento a través de tres ejercicios:

- Baile: Con percusión y melodía vocal se interpreta el Waltz Sobre las Olas de Juventino Rosas para trabajar la intimidad, el movimiento, la complicidad y evocar la risa haciendo movimientos exagerados.

- Movimiento con maracas: Se entrega una maraca y se toca un blues clásico de doce compases y se le cantan las indicaciones referidas a la orientación y lugar en el que se tiene que tocar el huevo. Este ejercicio favorece el movimiento de los brazos y trabaja la atención sostenida.

- Movimiento con el cuerpo: Se toca un blues de doce compases y se cantan las instrucciones referidas a una parte del cuerpo y el tipo de movimiento requerido cada dos o cuatro compases. Con esta paciente se prima el movimiento de la cabeza sobre el resto del cuerpo pidiéndole que mire al techo y baile con la cabeza al ritmo del blues.

- Improvisación con instrumento: Se realiza una improvisación de forma libre con cualquier instrumento que esté disponible. Este ejercicio está basado en el modelo de improvisación libre de Alvin (1975). Con llamadas de atención se intenta que la paciente no interrumpa la improvisación y mantenga la atención en la conducta de tocar.

- Interpretación de una canción específica: Se interpreta a dúo una canción creada específicamente para la paciente (Anexo 6). Esta canción tiene como objetivo que la paciente abra la boca y relaje la mandíbula.

- Improvisación vocal: Paciente y terapeuta improvisan con la voz. La forma musical resultante de la improvisación va en función del estado de ánimo del paciente y lo que este intenta expresar.

- Pasaje de despedida (Anexo 5): Se interpreta la canción de despedida. En la parte final de la canción la paciente tiene que estar atento para decir adiós dos veces. 


\section{RESULTADOS}

La puntuación general obtenida en el WAI-T fue de 209 sobre 252, puntuando 66 en "task", 82 en "bond" y 61 en "goal".

En la Tabla 2 se muestra la frecuencia de aparición de las conductas objetivo de cada uno de los tres factores que conforman la existencia de la alianza terapéutica en la sesión inicial final.

Tabla 2

Resultados descriptivos

\begin{tabular}{|c|c|c|c|c|c|c|}
\hline & \multicolumn{3}{|c|}{ Sesión inicial } & \multicolumn{3}{|l|}{ Sesión final } \\
\hline & $\begin{array}{l}\text { Aceptación } \\
\text { de los } \\
\text { objetivos }\end{array}$ & $\begin{array}{l}\text { Aceptación } \\
\text { de las } \\
\text { técnicas a } \\
\text { utilizar }\end{array}$ & $\begin{array}{l}\text { Creación } \\
\text { de un } \\
\text { lazo } \\
\text { afectivo }\end{array}$ & $\begin{array}{l}\text { Aceptación } \\
\text { de los } \\
\text { objetivos }\end{array}$ & $\begin{array}{l}\text { Aceptación } \\
\text { de las } \\
\text { técnicas a } \\
\text { utilizar }\end{array}$ & $\begin{array}{l}\text { Creación } \\
\text { de un lazo } \\
\text { afectivo }\end{array}$ \\
\hline $\begin{array}{l}\text { Frecuencia } \\
\mathrm{N}\end{array}$ & 3 & 30 & 6 & 13 & 64 & 22 \\
\hline $\begin{array}{l}\text { Frecuencia } \\
\text { A }\end{array}$ & 8 & 28 & 5 & 16 & 55 & 21 \\
\hline
\end{tabular}

En el anexo 7 es posible consultar las puntuaciones medias de respuesta de los participantes para cada ítem y factor.

\section{DISCUSIÓN}

Se ha realizado una búsqueda exhaustiva de estudios similares al presente, pero no se ha encontrado nada que integre musicoterapia, SD y AT. Como se mencionó antes, las investigaciones que relacionan SD y AT siguen una metodología muy distinta.

La presente investigación sienta precedente acerca de la importancia que tiene el desarrollo de la AT en la musicoterapia porque el elemento central en este tipo de terapia es la música y no la palabra. La comunicación emocional que se da entre el paciente y el musicoterapeuta se centra en la música, por tanto, sería irresponsable dar por supuesto el desarrollo de la AT sin someterlo a prueba. 
Este trabajo lanza una propuesta metodológica que puede ser determinante para realizar más investigaciones en el campo de la musicoterapia. La utilización de un software de codificación y procesamiento de datos es fundamental para estudios cualitativos y cuantitativos de musicoterapia porque en el presente no existen métodos estandarizados y validados de evaluación, por tanto, se deben utilizar herramientas como esta para adaptar el análisis de las técnicas existentes y de su impacto en los pacientes.

Como se ha podido comprobar, se han dado circunstancias que de haber sido de otra manera habrían dotado a esta investigación de mayor rigor científico. Resulta necesario replicar este trabajo incrementando el tamaño de la muestra, añadiendo un grupo control y llevando a cabo un instrumento para medir la AT adaptado a la población con SD. En relación con el instrumento es importante aclarar que el factor "Aceptación de los objetivos" del WAI no tiene una buena sinergia con algunas de las barreras de los pacientes con SD, por ejemplo la limitación de comprensión verbal (Nash y Heath, 2011).

\section{CONCLUSIONES}

Las puntuaciones obtenidas en el instrumento WAI-T, según el baremo, indican que existe relación terapéutica con ambos pacientes porque se encuentran por encima del percentil 75 . Se debe tener en cuenta que el WAI no es una prueba estandarizada y no está adaptada a pacientes con SD por lo que se debe proceder con cautela al interpretar esta medida. De facto, las investigaciones existentes que relacionan la AT con pacientes con SD son estudios cualitativos de caso.

Si se observan los estadísticos descriptivos de las frecuencias de aparición de las conductas favorables a la construcción de la AT (Anexo 7) se puede comprobar que hay un incremento considerable de conductas objetivo en la última sesión en comparación con la primera. Además del análisis a través de los estadísticos descriptivos se ha realizado un análisis no paramétrico de Wilcoxon con el fin de comprobar si la diferencia de las medias de respuesta de la primera sesión y la última son estadísticamente significativas (Anexo 8). Como era de esperar los niveles de significación obtenidos superaron ampliamente el valor necesario para establecer una diferencia significativa debido al escaso número de participantes en la investigación $(\mathrm{N}=2)$.

En conclusión resulta importante ahondar en el desarrollo y existencia de la AT dentro de la musicoterapia y comenzar un camino de investigación dirigido al rigor científico. Este 
camino debe nutrirse de datos objetivos, utilización de pruebas estandarizadas, replicabilidad de las intervenciones y sentido crítico.

\section{REFERENCIAS}

Alexander, L. B. y Luborsky, L. (1986). The Penn Helping Alliance Scales. The psychotherapeutic process: a research handbook. New York, NY: Guilford Press.

Alvin, J. (1975). Music Therapy. New York: Basic Books.

Andrade-González, N. y Fernández-Liria, A. (2016). Spanish Adaptation of the Working Alliance Inventory-Short (WAI-S). Current Psychology, 35, 169-177.

Ardito, R. B. y Rabellino, D. (2011). Therapeutic alliance and outcome of psychotherapy: historical excursus, measurements, and prospects for research. Frontiers in Psychology, 2, 270.

Bassler, M. y Nübling, R. (2015). Helping Alliance Questionnaire.

Beck, A. T. y Alford, B. A. (2009). Depression: Causes and Treatments (2nd ed.). Philadelphia: University of Pennsylvania Press.

Beck, A. T., Rush, A. J., Shaw, B. y Emery, G. (1979). Cognitive therapy of depression. New York, NY: Guilford Press.

Beck, A. T., Rush, A. J., Shaw, B. y Emery, G. (2005). Terapia Cognitiva de la Depresión. España: Editorial Desclée de Brower, S.A.

Bello, A., Onofrio, D. y Caselli, M. C. (2014). Nouns and predicates comprehension and production in children with Down syndrome. Research in Developmental Disabilities, 35, 761-775.

Bordin, E. S. (1979): The generalizability of the psychoanalytic concept of the working alliance. Psychotherapy: Theory, Research and Practice, 16(3), 252-260.

Bruscia, K. E. (1999). Modelos de improvisación en musicoterapia. Vitoria: Agruparte.

Carr, J. (1995). Down's syndrome: Children growing up. Cambridge: Cambridge University Press.

Chavin, M. (2002). Music as Communication. Alzheimer's Care Quarterly, 3(2), 145-156.

Duncan, B. L., Miller, S. D., Sparks, J. A., Claud, D. A., Reynolds, L. R., Brown, J. y Johnson, L. D. (2003). The Session Rating Scale: Preliminary Psychometric Properties of a "Working" Alliance Measure. Journal of Brief Therapy, 3, 1.

Flaig, N. K. y Large, E. W. (2014). Dynamic musical communication of core affect. Frontiers in Psychology, 5, 72.

Freud, S. y Breuer. J. (1955). Studies in hysteria, The standard edition of the complete psychological works of Sigmund Freud (Vol. 2). London: The Hogarth Press.

Groussard, M., Rauchs, G., Landeau, B., Viader, F., Desgranges, B., Eustache, F. y Platel, H. (2010). The neural substrates of musical memory revealed by fMRI and two semantic tasks. NeuroImage, 53, 1301-1309.

Gutiérrez, L. M. (2013). La Música Como Lenguaje y Medio de Comunicación. Ecos del Lejano Oriente en la Vanguardia Musical Orientalismo y Japonismo Musical. Entreculturas, 5, 1989-5097.

Harry, R. M., Purser, H. R. M. y Jarrold, C. (2012). Poor phonemic discrimination does not underlie poor verbal short-term memory in Down syndrome. Journal of Experimental Child Psychology, 115, 1-15. 
Hartmann, A., Orlinsky, D. y Zeeck, A. (2011). The Structure of Intersession Experience in Psychotherapy and Its Relation to the Therapeutic Alliance. Journal of Clinical Psychology, 67(10), 1044-1063.

Horvath, A. O. (1994). Empirical validation of Bordin's pantheoretical model of the alliance: the working alliance inventory perspective. The Working Alliance: Theory, Research and Practice, 109-128.

Horvath, A. O. y Greenberg, L. S. (1986). The development of the Working Alliance Inventory. The psychotherapeutic process: a research handbook. New York, NY: Guilford Press.

Hyde, K. L., Peretz, I. y Zatorre, R. J. (2008). Evidence for the role of the right auditory cortex in fine pitch resolution. Neuropsychologia, 46(2), 632-639.

Kay-Raining, E., Patricia, C., Denise, W., Pike, H. y Helmkay, A. (2008). Written and Oral Narratives of Children and Adolescents with Down Syndrome. Journal of Speech, Language, and Hearing Research, Vol. 51, 436-450.

Krupnick, J. L., Sotsky, S. M., Elkin, I., Watkins, J. y Pilkonis, P. A. (1996). The role of the therapeutic alliance in psychotherapy and pharmacotherapy outcome: Findings in the National Institute of Mental Health Treatment of Depression Collaborative Research Program. Journal of Consulting and Clinical Psychology, 64, 532-539.

Laws, G. y Bishop, D. V. M. (2003). A comparison of language abilities in adolescents with Down syndrome and children with Specific Language Impairment. Journal of Speech Language and Hearing Research, 46, 1324-1339.

Meichenbaum, D. (1987). Manual de inoculación de estrés. Barcelona: Ediciones Martínez Roca, S.A.

Michel, K. y Jobes, D. A. (2011). Building a Therapeutic Alliance with the Suicidal Patient. Washington: American Psychological Association.

Nash, H. y Heath, J. (2011). The role of vocabulary, working memory and inference making ability in reading comprehension in Down syndrome. Research in Developmental Disabilities, 32, 1782-1791.

Orlinsky, D. E., Ronnestad, M. H. y Willutzki, U. (2004). Fifty years of psychotherapy process-outcome research: Continuity and change. Bergin and Garfield's handbook of psychotherapy and behavior change, 5, 307-389.

Poeppel, D. y Hickok, G. (2004). Towards a new functional anatomy of language. Cognition, 92, 1-12.

Rigoulot, S., Wassiliwizky, E. y Pell, M. D. (2013). Feeling backwards? How temporal order in speech affects the time course of vocal emotion recognition. Frontiers in Psychology, 4, 367.

Robson, E. M. (1959). Language Music. Music Journal, 17(1), 9.

Roch, M., Florit, E. y Levorato, M. C. (2011). The advantage of reading over listening text comprehension in Down syndrome: What is the role of verbal memory? Research in Developmental Disabilities, 33, 890-899.

Roizen, N. J. (1997). Down syndrome in M. L. Batshaw, Children with disabilities (4th ed.). Baltimore: Brookes.

Roth, A. y Fonagy, P. (2005). What works for whom: A critical review of psychotherapy research (2nd ed.). New York: Guilford Press.

Ruiz, A., Díaz, M. y Villalobos, A. (2012). Manual de Técnicas de Intervención Cognitivo Conductual. España: Desclée De Brouwer, S.A.

Safran, J. D. y Wallner, L. K. (1991). The Relative Predictive Validity of Two Therapeutic Alliance Measures in Cognitive Therapy. A Journal of Consulting and Clinical Psychology, 3(2), 188-195. 
Schön, D.,Gordon, R.,Campagne, A.,Magne, C.,Astésano, C., Anton, J. L. y Besson, M. (2010). Similar cerebral networks in language, music and song perception. NeuroImage, 51,450-461.

Spreckelmeyer, K. N., Altenmüller, E. O., Colonius, H. y Münte, T. F. (2013). Preattentive processing of emotional musical tones: a multidimensional scaling and ERP study. Frontiers in Psychology, 4, 656.

Summers, R. F. y Barber, J. P. (2003). Therapeutic Alliance as a Measurable Psychotherapy Skill. Academic Psychiatry, 27, 3.

Toll, S. (2007). Communication through music. Alzheimer's \& Dementia, 3(3), S173.

Vázquez, M. (2001). Técnicas de relajación y respiración. Madrid: Síntesis.

Warlick, C., Richter, K. P., Catley, D., Gajewski, B. J., Martin, L. M. y Mussulman, L. M. (2018). Two brief valid measures of therapeutic alliance in counseling for tobacco dependence. Journal of Substance Abuse Treatment, 86, 60-64.

Wilson, S. J., Abbott, D. F., Tailby, C., Gentle, E. C., Merrett, D. L. y Jackson G. D. (2013). Changes in singing performance and fMRI activation following right temporal lobe surgery. Cortex, 49, 2512-2524.

Wilson, S. J., Abbott, D. F., Lusher, D., Gentle, E. C. y Jackson, G. D. (2011). Finding your voice: A singing lesson from functional imaging. Human Brain Mapping, 32(12), $2115-2130$. 